\title{
Principes, applications et perspectives de l'imagerie radiologique numérique
}

L'angiographie numérique est la principale application de la numérisation de l'imagerie radiologique. Par voie veineuse, elle permet notamment d'explorer les vaisseaux de gros et moyen calibre. Par voie artérielle, elle va progressivement remplacer l'artériographie traditionnelle.

\section{Pascal Lacombe Danièle Verderi Michel Mouvier Daniel W. Foster Pierre Petitbon Victor Bismuth}

\section{ADRESSE}

P. Lacombe, D. Verderi, M. Mouvier, D. W. Foster, P. Petitbon, V. Bismuth :

Service de radiologic, hôpital Ambroise-Paré, 92100 Boulogne. a numérisation de l'imagerie radiologique a débuté dans les années i970. Elle s'est appliquée initialement à la médecine nucléaire. Son développement s'est effectué parallèlement aux progrès de l'informatique. L'apparition d'ordinateurs à haute capacité mémoire et de programmes informatiques plus élaborés a permis la numérisation et le traitement d'autres modes d'imagerie plus complexes comme la tomodensitométrie, l'angiographie numérisée, l'échographie et l'imagerie par résonance magnétique. En I 985 , environ $30 \%$ de l'imagerie médicale est numérisée et son accroissement est constant. Nous nous proposons de revenir sur le mode de formation de l'image numérisée standard et son application à l'angiographie numérique. Nous envisagerons ensuite la valeur, les indications de cette technique, ainsi que ses perspectives.

\section{Numérisation de l'image radiologique standard}

La chaîne de numérisation du signal vidéo vient s'implanter sur une chaîne radiologique préexistante, comportant un amplificateur de luminance. Cette chaîne est compo- sée d'une caméra vidéo, d'un convertisseur analogique-numérique, d'un ordinateur et d'un écran de télévision, permettant la visualisation et la reproduction des images.

La numérisation de l'image se fait de la façon suivante : après avoir traversé la région anatomique à explorer, l'image radiante est transformée en image visible par l'amplificateur de luminance. La caméra vidéo, couplée à l'amplificateur de luminance, est munie d'un tube analyseur. Ce dernier balaye la surface de l'amplificateur de luminance et transmet un signal photo-électrique directement proportionnel à la luminosité de chaque point de la surface.

La surface analysée est découpée en petits éléments de surface appelés pixels. Le nombre de pixels utilisé pour analyser l'image définit la matrice. Les matrices les plus couramment utilisées comportent $512 \times 512$ pixels. A l'heure actuelle, apparaissent de nouvelles matrices améliorant la définition de l'image ( $1024 \times$ 1024).

Le signal électrique fourni par la caméra va être numérisé grâce à un convertisscur analogique-numérique. Le processus de numérisation se fait en langage binaire selon une échelle de valeur exprimée en bits 
qui détermine la p̄rofondeur de numérisation. Cette prof ondeur de numérisation détermine elle-même l'échelle des valeurs de gris; par exemple, une profondeur de 8 bits correspond en fait à 2 puissance 8 niveaux de gris c'est à dire 256 valeurs. On voit ainsi que la numérisation consiste finalement à transformer une information lumineuse continue, ou analogique, (signal vidéo), en une série de valeurs discontinues où un double processus intervient : organisation de l'image selon une matrice, et attribution d'une valeur numérique au niveau de chaque pixel.

Toutes ces informations sont stockées dans l'ordinateur. A partir de ces données chiffrées, l'ordinateur reproduit l'image sur un écran de télévision grâce à un convertisseur numérique-analogique.

\section{Radiographie standard numérisée}

Le développement de l'imagerie radiologique numérisée standard est à l'heure actuelle beaucoup moins important que celui de l'angiographie numérique. Les utilisateurs de ce type d'appareil se sont en effet heurtés à la taille réduite de l'amplificateur de luminance et à la résolution spatiale insuffisante pour l'étude des petites structures anatomiques. Le principal avantage de l'image radiologique numérique standard est celui de la réduction de la surface sensible utilisée. Il est en effet possible de reproduire sur un film de très petit format l'image numérique reproduite sur l'écran de télévision. L'apparition de la matrice r 024 et d'amplificateurs de luminance de plus grande taille qui peuvent explorer l'ensemble de l'abdomen ou des deux champs pulmonaires permettra sans doute le véritable développement de cette technique qui, par la réduction des surfaces sensibles, pourrait entraîner à l'avenir une diminution des coûts de santé.

\section{Angiographie numérisée par soustraction}

Le principe de l'angiographie numérisée est d'effectuer une séquence d'images numérisées sous- traites, pixel par pixel, à partir d'une image de référence appelée masque. Si au cours de la séquence, aucune modification dans la luminosité de chaque point ne survient, l'image résultante de la soustraction sera uniformément grise. Si à l'inverse, on injecte par voie intraveineuse un produit de contraste iodé, dès que ce produit de contraste apparaît au niveau d'une structure vasculaire artérielle la valeur des pixels, en superposition du vaisseau, va se modifier et ce vaisseau apparaîtra sur l'image de soustraction. Le but de la soustraction est en fait d'éliminer tous les éléments qui restent invariables au cours de la séquence.

Comme pour une image de tomodensitométrie, il est possible, puisqu'il s'agit d'une image numérique, de modifier la luminosité de l'image résultante, de la rendre plus claire ou plus foncée (changement de niveau). De même, il est possible d'augmenter le contraste relatif d'une structure vasculaire par rapport à son environnement. Cette fonction de fenêtrage permet ainsi la détection de vaisseaux faiblement opacifiés.

Une fois que la séquence angiographique est réalisée, il est possible de travailler les images avec l'ordinateur et de les reproduire. Le traitement différé se fait selon des programmes informatiques plus ou moins élaborés. Le premier impératif du traitement de l'image est celui du choix de l'image de référence (remasquage). Chaque image de la séquence peut, isolément ou en association avec d'autres, être utilisée comme image de masque. On peut même utiliser l'image de meilleure opacification vasculaire. Il est actuellement possible d'effectuer un remasquage synchronisé à l'électrocardiogramme de façon à compenser le mouvement des structures cardio-vasculaires.

Le deuxième impératif du traitement de l'image est celui de la compensation des mouvements intempestifs qu'a pu présenter le patient au cours de la séquence. Chaque mouvement risque en effet d'altérer la qualité de la soustraction. A partir de l'image jugée optimale, on peut toutefois effectuer des corrections par recalage manuel ou automatique du masque. Ces recalages peuvent se faire verticalement, transversalement, mais également par correction de rotation.

Il va sans dire que les mouvements intempestifs ne sont pas tous compensables par l'ordinateur et ils sont en grande partie responsables des échecs de l'angiographie numérisée intraveineuse.

\section{Angiographie \\ intraveineuse}

Les possibilités de rehaussement des contrastes grâce à la soustraction et au remasquage permettent en fait une appréciation morphologique des vaisseaux faiblement opacifiés. Les premières angiographies numériques ont été pratiquées à partir d'injections intraveineuses effectuées, soit au niveau d'une veine périphérique ( $p l i$ du coude), soit au niveau d'une veine centrale (veine cave supérieure ou veine cave inférieure). Le produit de contraste est injecté à un débit élevé (I 5 à 20 $\mathrm{ml} / \mathrm{s}$ ). Pour la grande majorité des territoires artériels, il faut injecter environ $40 \mathrm{ml}$ de produit de contraste par site anatomique. La qualité de l'angiographie numérique intraveineuse est directement conditionnée à la coopération du patient (immobilité totale) et à la qualité de ses fonctions hémodynamiques. Chez un patient ayant présenté un ou plusieurs infarctus, ayant des antécédents d'insuffisance cardiaque ou une arythmie complète, il est illusoire d'obtenir une imagerie satisfaisante par angiographie intraveineuse. Dans ces cas il convient d'emblée d'envisager l'utilisation de la voie artérielle.

Quelques accidents ont été signalés dans certaines séries (œdème pulmonaire, ou poussée hypertensive, ou poussée d'insuffisance coronarienne). Ces complications sont rares mais méritent d'être prévenues en adaptant la technique angiographique à l'état du patient.

Le risque lié à l'utilisation de quantités importantes de produit de contraste chez des patients explorés en ambulatoire doit être souligné. Au moment de la prise du rendez-vous, il nous paraît important de préciser les antécédents du patient, de demander une exploration des fonc- 
tions rénales, de dépister des antécédents d'allergie ou de diabète afin de prévenir ce risque.

\section{Angiographie artérielle numérique}

Toutes les techniques de cathétérisme artériel avec l'injection globale, sélective voire hypersélective, sont applicables en angiographie artérielle numérique. L'utilisation de cathéters de très petit calibre $(5$ voire $4 \mathrm{~F}$ ) et de certaines voies d'abord artérielles jusque-là peu usitées (voie humérale rétrograde) rendent ce type d'exploration moins invasif que les techniques conventionnelles. Si l'angiographie artérielle numérique a une résolution spatiale moindre que l'artériographie conventionnelle, à l'inverse sa résolution en contraste est très supérieure. Grâce à la possibilité de rehaussement du contraste, on utilise fréquemment par voie artérielle un produit de contraste dilué permettant une baisse de l'osmolalité du produit injecté et une meilleure tolérance par le patient. En raison de la qualité de l'opacification lors des injections globales, la fréquence des opacifications sélectives a nettement diminué, en particulier dans la pathologie athéromateuse. Certaines équipes, proposent aujourd'hui ce type d'exploration sans hospitalisation, en raison de la bonne tolérance de l'examen et du petit calibre du cathéter utilisé par voie humérale.

\section{Étude des}

vaisseaux pulmonaires

4. Chilcote WA, Modic MT, Pavlicek WA, et al. Digital subtraction angiography of the carotid arteries: a comparative study of 100 patients. Radiology 1981; 139: 287-95.

5. Crummy AB, Stieghorst MF, Strother CM Digital subtraction angiography: current status and use of intra-arterial injection. Radiology 1982; 145: 303-7.

6. Engels PHC, Ludwig JW, Verhoeven LAJ. Left ventricule evaluation by digital video subtraction angiocardiography. Radiology 1982; 144: 471-4.

7. Frija G, Lacombe P, Bacourt F, et al. Evaluation de la chirurgie vasculaire par angiographie numérisée; étude de 127 patients. Ann Radiol (Paris) 1983; 26: 589-93.

8. Hoffman MG, Gomes AS, Pais SO. Limitations in the interpretation of intravenous carotid digital subtraction angiography. $A 7 R$ 1984; 142: digital
L'angiographie pulmonaire numérique est de réalisation technique difficile en raison de la très grande mobilité des structures vasculaires au niveau pulmonaire et de leur contiguïté avec les structures cardiaques mobiles. L'utilisation d'une cadence rapide, voire d'une synchronisation à l'ECG permet de pallier ces inconvénients.

Dans la maladie thrombo-embolique, les injections périphériques, ou au niveau de la veine cave permettent une étude de bonne qualité des artères tronculaires, lobaires, segmentaires, voire sous-segmentaires, dans environ $90 \%$ des cas. Cet examen est parfaitement toléré, en particulier dans les embolies pulmonaires massives où le caillot est facilement visible dans les gros troncs pulmonaires. A l'inverse, si on est confronté au diagnostic de l'embolie pulmonaire de très petite taille (données de la scintigraphie), il convient de réaliser d'emblée une injection sélective, voire hypersélective pulmonaire. A l'heure actuelle, l'angiographie numérisée pulmonaire, qu'elle soit sélective ou non sélective, est le seul type d'imagerie vasculaire pulmonaire que nous utilisons (figure I).

\section{Exploration}

de l'aorte thoracique

$\mathrm{Si}$ les conditions hémodynamiques du patient sont bonnes, nous effectuons cette exploration exclusivement par voie veineuse dans les maladies chroniques de l'aorte thoracique (anévrisme, anomalies congénitales, dissection chronique, aortite inflammatoire).

Au cours des dissections aiguës de l'aorte thoracique, plus particulièrement s'il existe une insuffisance aortique ou un bas débit cardiaque, nous préférons l'utilisation de la voie artérielle d'emblée, afin d'apprécier l'importance du reflux aortique, l'atteinte éventuelle des artères coronaires ainsi que l'extension précise au niveau des vaisseaux du cou (figure 2).

\section{Vaisseaux cervico-encéphaliques}

Nous adaptons notre technique d'opacification aux résultats de l'examen doppler et de l'échotomographie haute résolution des carotides (figures 3 et 4). S'il existe une lésion significative au vu de ces examens, l'angiographie numérisée intraveineuse est suffisante pour la mettre en évidence et apprécier l'état du lit distal carotidien. Dans $95 \%$ des cas, aucune opacification artérielle complémentaire n'est effectuée avant l'intervention chirurgicale. A l'inverse, si l'examen échotomographique doppler des carotides est normal, nous effectuons d'emblée une opacification artérielle numérique par injection dans l'aorte ascendante d'un produit de contraste dilué. En ce qui 


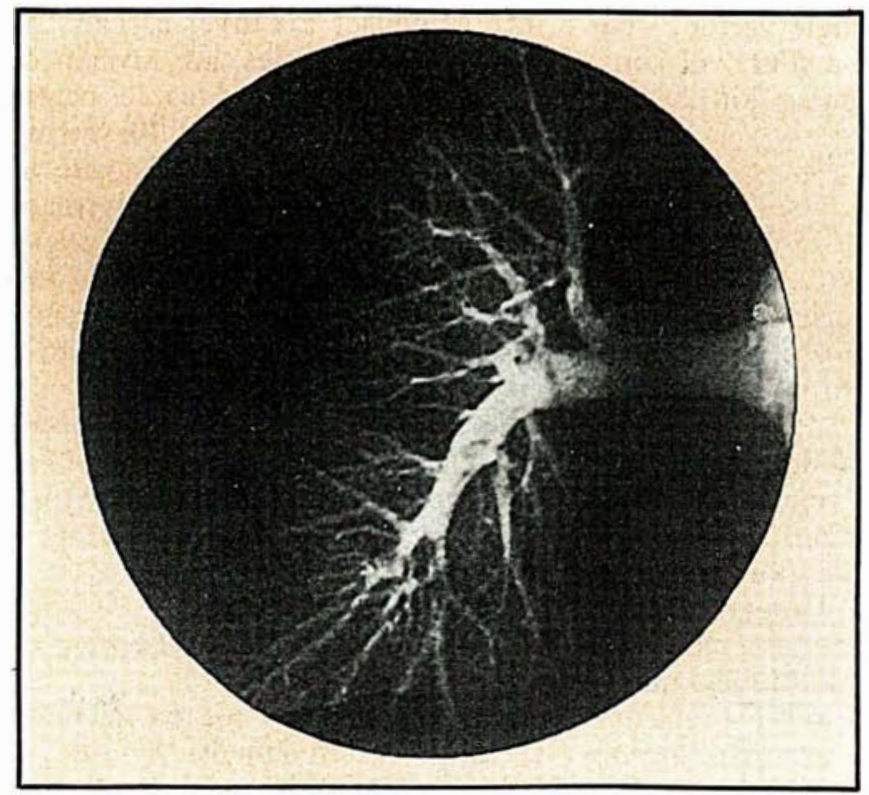

Figure 1. Angiographie pulmonaire numérique par injection au niveau de la veine cave. Centrage sur le champ droit. Présence de multiples.lacunes dans le tronc de l'artère lobaire inférieure droite et dans chacune des branches de la pyramide basale. Embolie de gravité moyenne.

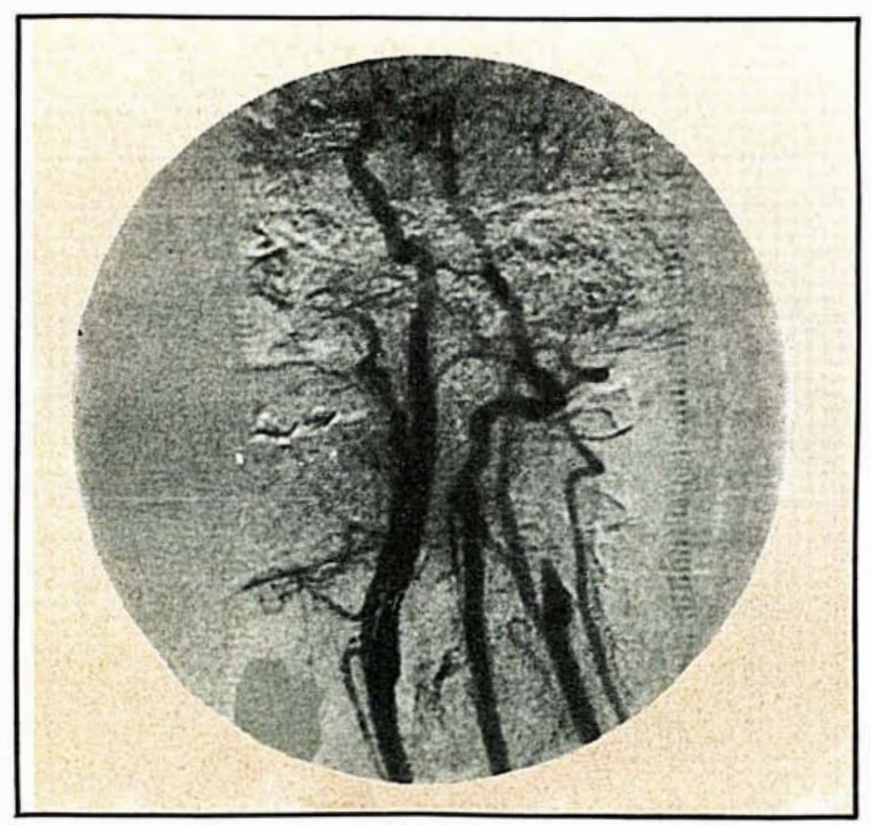

Figure 3. Exploration carotidienne par angiographie intra-veineuse. Centrage au niveau du cou. Il existe une occlusion complète de l'artère carotide interne gauche associée à une sténose non significative à l'origine de l'artère carotide externe.

$\mathrm{m} / \mathrm{s} n^{0} 3$ vol. 2 mars 86

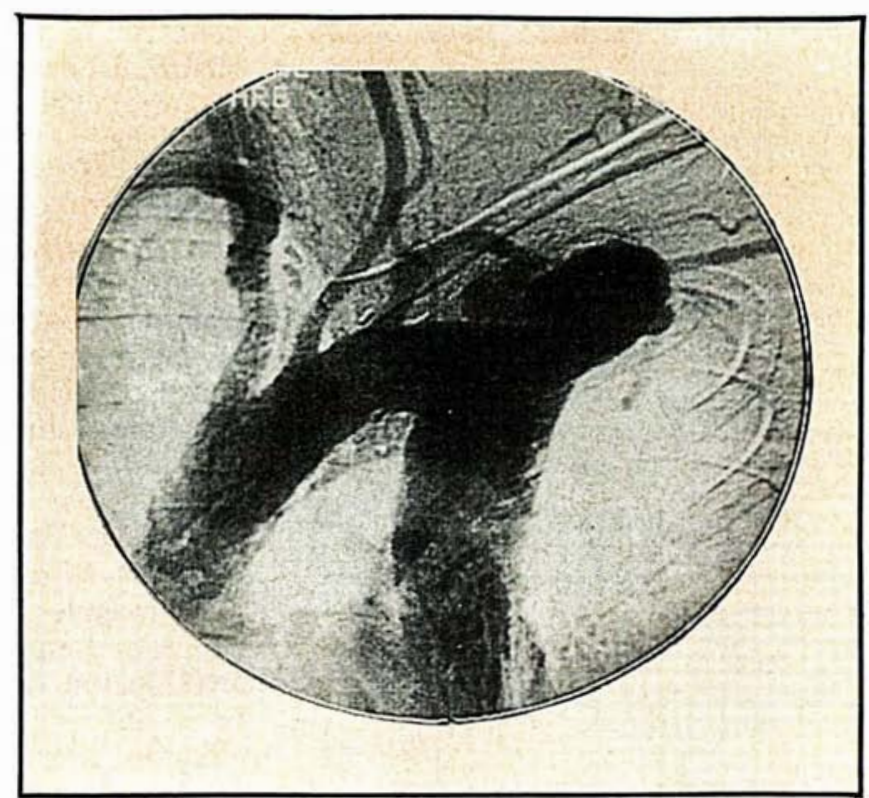

Figure 2. Anévrisme post-sousclavier de l'aorte thoracique. Incidence en oblique antérieure gauche. L'angiographie intra-veineuse permet de préciser le siège de l'anévrisme par rapport aux pieds de l'artère sous-clavière gauche. Aucune autre exploration angiographique n'a été nécessaire avant l'intertention chirurgicale.

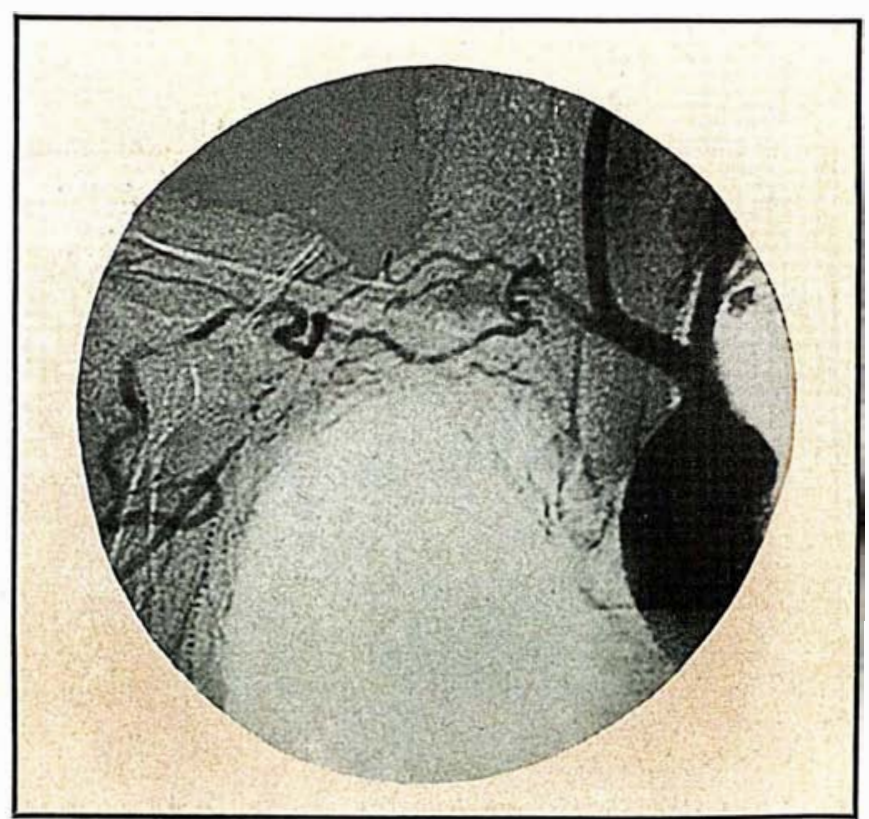

Figure 4. Gerbe aortique en oblique antérieure droite. Exploration par voie veineuse. Il existe une occlusion complète de l'artère sous-clavière droite et de l'artère axillaire. Revascularisation de l'artère humérale par une volumineuse branche collatérale. Aortite inflammatoire non spécifique. 


\section{RÉFÉRENCES}

9. Modig MT, Weinstein MA, Starnes DL, et al. Intravenous digital subtraction angiography of the intracranial veins and dural sinuses. Radiology 1983; 146: 383-9.

Io. Pariente D, Ernest C, Lacombe P, Lallemand D, Frija G. L'angiographie numérisée : applications pratiques en pédiatrie. Ann Pediat (Paris) 1983; $30: 175-81$.

11. Pond GD, Ovitt TW, Capp MP. Comparison of conventional pulmonary angiography with intravenous DSA for pulmonary embolic disease. Radiology 1983; 147: 345-50.

12. Reilley RF, Smith CW, Price RR, et al. Digital subtraction angiography: limitations for the detection of pulmonary embolism. Radiology 1983; 149: 379-82.

13. Seurot M, Sirinelli D, Coombel B, et al. Angiograjphie numérique dans l'exploration de l'hypertension artérielle. 7 Radiol 1983; 64: 495-9.

14. Smith CW, Winfield AC, Price RR, et al. Evaluation of digital venous angiography for the diagnosis of renovascular hypertension. Radiology 1982; 144: 5 I-4.

15. Wood GW, Lukin RR, Tomsick TA, Chambers AA. Digital subtraction angiography with intravenous injection: assessment of $\mathrm{r} 000$ carotid bif urcations. $A 7 R$ 1983; 140: 855-9. concerne la pathologie vertébro-basilaire, à l'exception d'un vol sousclavier, l'exploration se fait par voie artérielle.

\section{Aorte \\ abdominale}

Comme pour l'aorte thoracique, l'exploration de l'aorte abdominale dans les maladies chroniques, se fait par voie veineuse. Pour les anévrismes de l'aorte abdominale, l'exploration par voie veineuse permet d'apprécier l'extension de l'anévrisme et son siège par rapport aux artères viscérales. Si l'anévrisme de l'aorte abdominale est associé à une artériopathie chronique des membres inférieurs, nous préférons effectuer d'emblée une exploration par voie artérielle pour opacifier l'ensemble des axes en amont de l'anévrisme et jusqu'aux extrémités des membres inférieurs (figure 5).

L'étude des artères rénales proximales est pratiquement toujours de bonne qualité par angiographie intraveineuse; à l'inverse, l'analyse des artères rénales au niveau du sinus du rein, ou dans le parenchyme rénal est difficile. De même, il est souvent difficile d'apprécier l'extension distale d'une dysplasie artérielle rénale. Ainsi, l'angiographie intraveineuse reste un excellent mode de dépistage des lésions proximales. Si une lésion est mise en évidence, une exploration par voie artérielle est souvent nécessaire pour juger des possibilités thérapeutiques (intervention chirurgicale, angioplastie transluminale).

\section{Artériopathies des membres inférieurs}

Dans notre expérience, en raison de la taille relativement réduite de notre amplificateur de luminance, nous n'explorons pas cette pathologie par voie veineuse; il serait en effet nécessaire d'effectuer un nombre très important d'injections de produit de contraste. Cette technique par voie veineuse est
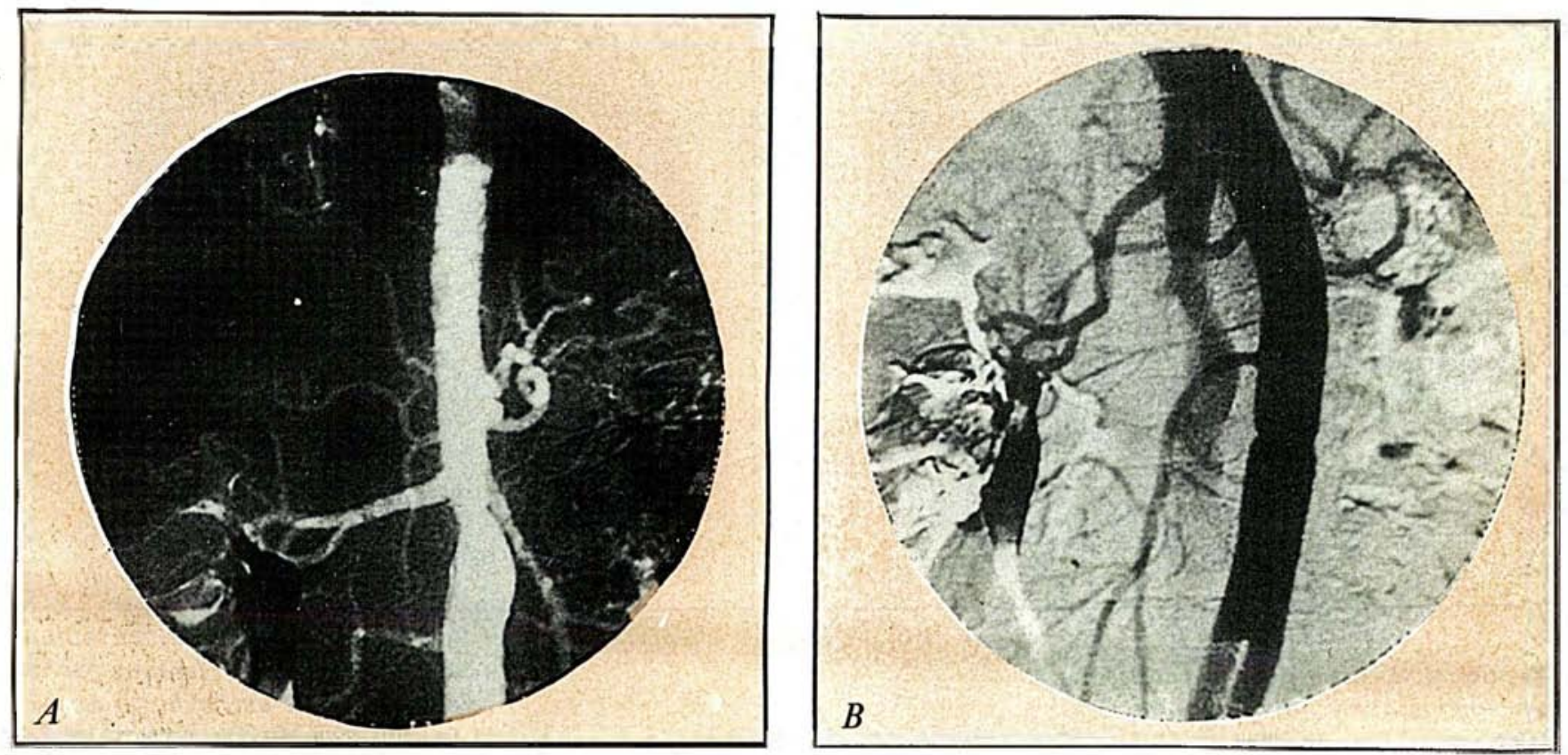

Figure 5. Maladie de Takayasu. Angiographie intra-veineuse. A. Sténose sous-rénale de l'aorte abdominale suivie d'une dilatation post-sténotique calcifiée. Agénésie rénale gauche. B. Contrôle post-opératoire. Prothèse aorto-bi-iliaque. L'artère rénale droite, ainsi que l'artère mésentérique supérieure ont été directement réimplantées sur la prothèse. 


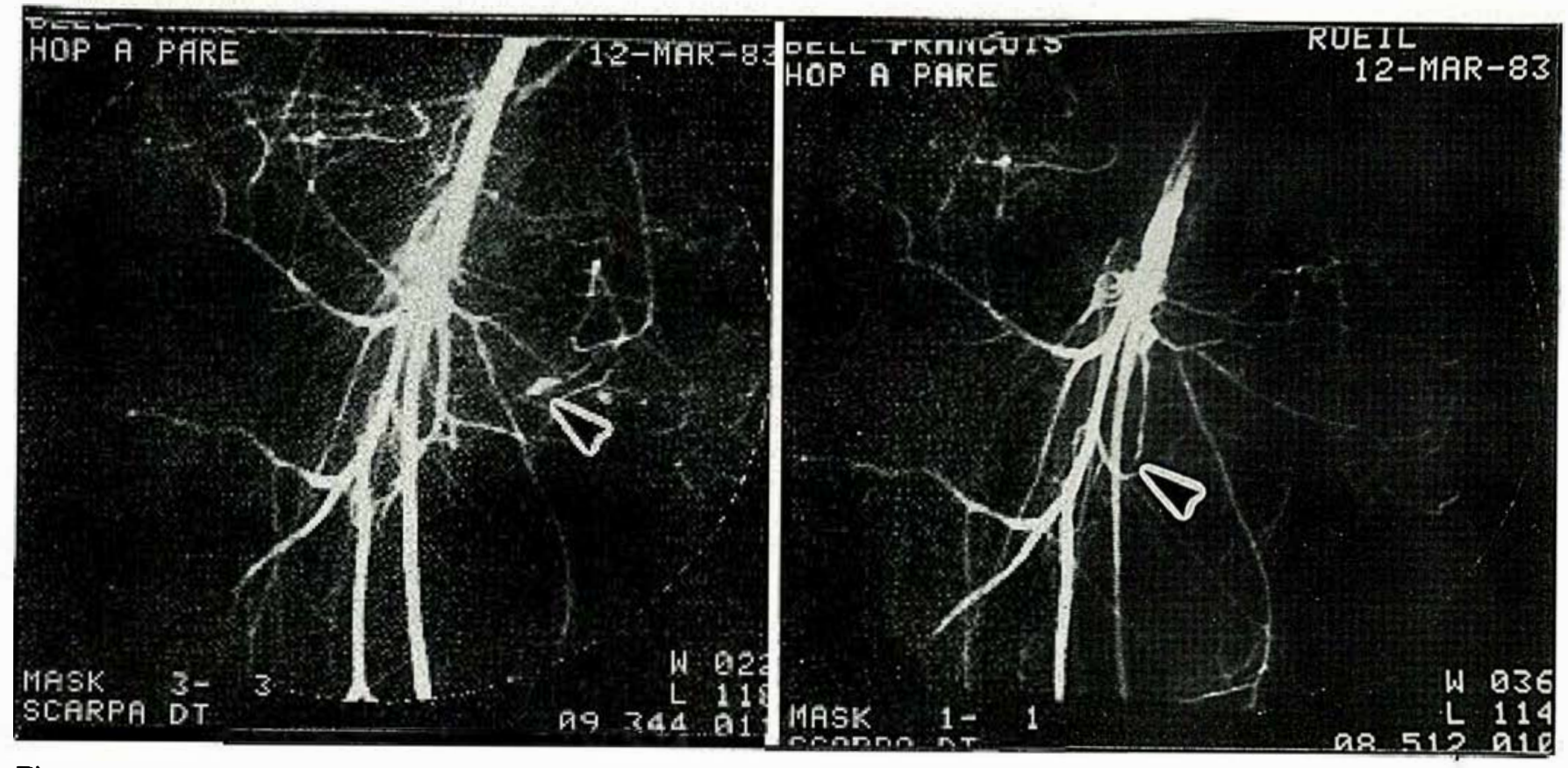

Figure 6. Traumatisme pelvien majeur. Hématome extensif de la cuisse. Ligature opératoire des deux artères hypogastriques sous clampage aortique temporaire. A gauche : angiographie artérielle numérique centrée sur le triangle de Scarpa droit. Présence de multiples zones d'extravasation vasculaire traduisant une rupture artérielle (flèche). A droite : contrôle après embolisation. L'artère alimentant les zones d'extravasation est occluse (flèche).

néanmoins envisageable lorsqu'on dispose d'un amplificateur de luminance de grande taille. Cependant, la qualité de l'imagerie est parfois aléatoire au niveau des axes de jambe et surtout de la cheville. En revanche, pour les contrôles postopératoires de chirurgie vasculaire, une exploration par voie veineuse donne d'excellents résultats, quel que soit le site anatomique.

\section{Pathologie \\ ostéo-articulaire}

Ici, nous utilisons presque toujours la voie artérielle. En cas de traumatisme aigu, avec rupture artérielle, le dépistage des extravasations est facilité par la soustraction, après une injection globale de produit de contraste (figure 6). Dans la pathologie tumorale, en partictilier en cas de processus tumoral hypervascu-

$\mathrm{m} / \mathrm{s} n^{\circ} 3$ vol. 2 mars 86 laire, ou modérément vascularisé, l'angiographie artérielle numérique permet le diagnostic de localisation et d'extension. Cette technique prend toute sa valeur dans les zones où l'examen tomodensitométrique est moins performant (creux poplité, extrémité).

\section{Pédiatrie}

Les explorations se font chez l'enfant, le nourrisson, voire même chez le nouveau-né sous prémédication légère, très rarement sous anesthésie genérale. Les champs d'application de cette technique sont les suivants : exploration des vaisseaux pulmonaires dans les bronchopathies sévères ou les séquelles de pneumopathies infectieuses, étude des arcs vasculaires anormaux, des artères rénales et enfin contrôle des transplantations rénales.

\section{Exploration cardiaque et coronarienne}

L'étude des fonctions ventriculaires gauches par angiographie intraveineuse donne des résultats fiables, comparables à ceux de l'exploration par injection intra-ventriculaire. En revanche, pour l'exploration des artères coronaires, la technique par voie veineusè est insuffisante. Chez les valvulaires, les artères coronaires sont analysables par simple injection non sélective sus-sigmoïdienne. Il en est de même pour le contrôle systématique des pontages aorto-coronariens.

A l'inverse, lors du premier examen chez un coronarien, les injections sélectives sont souvent nécessaires mais l'exploration numérique n'a pas encore fait la preuve de sa supériorité par rapport aux explorations conventionnelles en radio-cinéma. 


\section{TIRÉS A PART}

P. Lacombe : Service de radiologic, hôpital Ambroisc-Paré, 92100 Boulogne.
Toutes les techniques qui viennent d'être décrites ne permettent en fait qu'une appréciation morphologique vasculaire. Or, à partir de ces données chiffrées il est possible de faire des études dites fonctionnelles ou paramétriques: (a) étude des variations dans le temps des valeurs numériques au niveau de certains pixels pour évaluer la cinétique du produit de contraste dans les vaisseaux; cette première modalité va permettre l'évaluation objective des débits relatifs au niveau des différents systèmes artériels; (b) étude dans l'espace des variations d'atténuation des pixels, qui va permettre de quantifier en fonction des données vidéo-densitométriques, les rétrécissements vasculaires et d'effectuer également des contourages automatiques vasculaires ou d'autres cavités (ventricule gauche). Ces études paramétriques sont encore du domaine de l'expérimentation mais les résultats préliminaires sont prometteurs

\section{Summary}

Digital subtraction angiography (DSA) is the main application of digitalization for the time being. Intravenous DSA is an accurate and safe procedure for the visualisation of large and medium size vessels. Its quality is related to the cardiac output, to the cooperation of the patient, to the performance of the software, the radiological set and the experience of the phycician. Intra-arterial DSA has an increasing efficiency in the investigation of medium size and tiny vessels. It is safer than conventional arteriography, because of the possibility of using diluted contrast medium and smaller catheters. In the future, parametric studies will open new fields for evaluation of flow and arterial stenosis. 